\title{
Diastolic mechanisms of impaired exercise tolerance in aortic valve disease
}

\author{
P J OLDERSHAW, K D DAWKINS, D E WARD, D G GIBSON \\ From the Cardiac Department, Brompton Hospital, London
}

SUMMARY In order to determine the significance of abnormalities of diastolic function in patients with left ventricular hypertrophy, exercise echocardiography to heart rates of 140 to 150 beats $/ \mathrm{min}$ was performed in 18 normal subjects and 14 patients after aortic valve replacement. Simultaneous echo-, phono-, and electrocardiograms were recorded. Left ventricular cavity size was determined at end-diastole and end-systole. The timing of mitral valve opening and closure was measured, and hence left ventricular filling time derived, expressed either as $\mathrm{ms} /$ beat, or $\mathrm{s} / \mathrm{min}$ when multiplied by heart rate. Isovolumic relaxation was taken as the interval between A2 and mitral valve opening. Systolic function, assessed from cavity dimensions, peak VCF, and QA2 interval was normal in all but two patients at rest and on exercise. Isovolumic relaxation was prolonged at rest in the patients to $85 \pm 8 \mathrm{~ms}$ (normal $69 \pm 9 \mathrm{~ms}$ ), but left ventricular filling times were normal. With exercise, in normal subjects, isovolumic relaxation remained constant, but filling times dropped strikingly from $380 \pm 66 \mathrm{~ms} /$ beat, or $27 \pm 2 \mathrm{~s} / \mathrm{min}$ at rest to $115 \pm 10 \mathrm{~ms} /$ beat or $16 \pm 2 \mathrm{~s} / \mathrm{min}$. In patients with left ventricular hypertrophy, isovolumic relaxation dropped on exercise to $41 \pm 15 \mathrm{~ms}$. Filling periods were normal at rest, $367 \pm 67 \mathrm{~ms} /$ beat or $27 \pm 3 \mathrm{~s} / \mathrm{min}$, but failed to show the normal drop with exercise, being $240 \pm 44 \mathrm{~ms} /$ beat or $28 \pm 4 \mathrm{~s} / \mathrm{min}$. At heart rates above $120 / \mathrm{min}$, separation between the two groups was complete.

Thus, striking abnormalities of left ventricular filling can be demonstrated on exercise in patients with left ventricular hypertrophy. They appear to represent loss of mechanisms whereby rapid diastolic filling is achieved in the normal subject.

Numerous studies of left ventricular diastolic function have been performed in man at rest, both in normal subjects and in patients with disease. Several techniques, including haemodynamic, ${ }^{1}$ ultrasound, ${ }^{2}$ and radionuclide $^{3}$ have all confirmed a characteristic pattern of volume change consisting of an early period of rapid filling, a mid-diastolic period of diastasis, and a further volume increase during atrial systole. In patients with left ventricular hypertrophy, this pattern is significantly modified. Isovolumic relaxation is prolonged, ${ }^{4}$ peak rate of dimension increase during filling is reduced, ${ }^{5}$ and, in late diastole, passive stress strain relations may be abnormal. ${ }^{6}$ It was the purpose of the present study to examine early diastolic events in a group of such patients during exercise, and to compare the findings with normal. We have therefore determined a series of diastolic time intervals using echo- and phonocardiography in order to investigate

Accepted for publication 8 February 1983 the manner in which the abnormal resting filling pattern is modified during exercise in those with left ventricular hypertrophy.

Patients and methods

Observations were made on 14 patients studied five to 26 months after aortic valve replacement. Operation was for dominant aortic stenosis in 12, and aortic regurgitation in two. Their ages ranged from 50 to $68 \mathrm{\omega}$ years; seven had Björk-Shirley prostheses and seven $\underset{2}{-}$ Starr-Edwards prostheses. All were in sinus rhythm, $\bullet$ and none was taking any treatment other than warfa- $\bar{D}$ rin. As a control series, 18 normal subjects, aged 20 to $\stackrel{\odot}{?}$ 32 , were used. None had clinical evidence of any heart $\underline{T}$ disease. The patients had previously undergone a maximum symptom limited formal treadmill exercise $\stackrel{\mathbb{\Omega}}{\triangle}$ test, using the Bruce protocol. All were able to reach $\stackrel{\mathbb{Q}}{\Omega}$ $97 \pm 7 \%$ of maximum predicted heart rate for age and sex. Exercise tolerance, however, was limited to 
$63 \pm 21 \%$ of predicted normal for age and sex. Five additional patients were studied during a clinically indicated electrophysiological study. Of these, two proved to have normal hearts, two had ventricular tachycardia, and one had Wolff-Parkinson-White syndrome.

Patients were initially studied at rest, in the left semilateral position. Simultaneous echo-, phono, and electrocardiograms were recorded using a Cambridge CDS system, operating at a paper speed of $100 \mathrm{~mm} / \mathrm{s}$. Subjects were then exercised sitting, using a method based on that of Corallo et al., ${ }^{7}$ inclined to the left, with their legs approximately horizontal, using an Elema-Schonander bicycle ergometer. Exercise was started at a level of $150 \mathrm{kpm} / \mathrm{min}$, and was increased at two minute intervals until a heart rate of 140 to $150 /$ min was reached. Recordings were made at peak exercise, and at intervals during recovery as the heart rate slowed. In patients undergoing electrophysiological studies observations were obtained at rest, and at the end of a two minute period of right atrial pacing at a rate of $150 / \mathrm{min}$ (RR interval $400 \mathrm{~ms}$ ).
Resting M-mode echocardiograms were recorded to show left ventricular cavity size and posterior wall and septal thickness continuously throughout the cardiac cycle. In addition, mitral valve echoes were recorded from the level at which complete coaptation of anterior and posterior leaflets was observed. During exercise or atrial pacing, mitral leaflet echoes were recorded in all patients, and, when possible, left ventricular cavity size (Fig. 1). A phonocardiogram was recorded from the aortic area using a Leatham suction microphone and a medium or high frequency cut-off filter.

From these records, the following measurements were made:

(1) RR interval and heart rate.

(2) End-diastolic and end-systolic dimensions, synchronous with the $Q$ wave of the electrocardiogram and $\mathrm{A} 2$, respectively, at rest, and at peak exercise. Shortening fraction was derived as systolic reduction in dimension divided by end-diastolic dimension.

(3) QA2 interval, A2 being taken as the closure sound of the prosthesis.
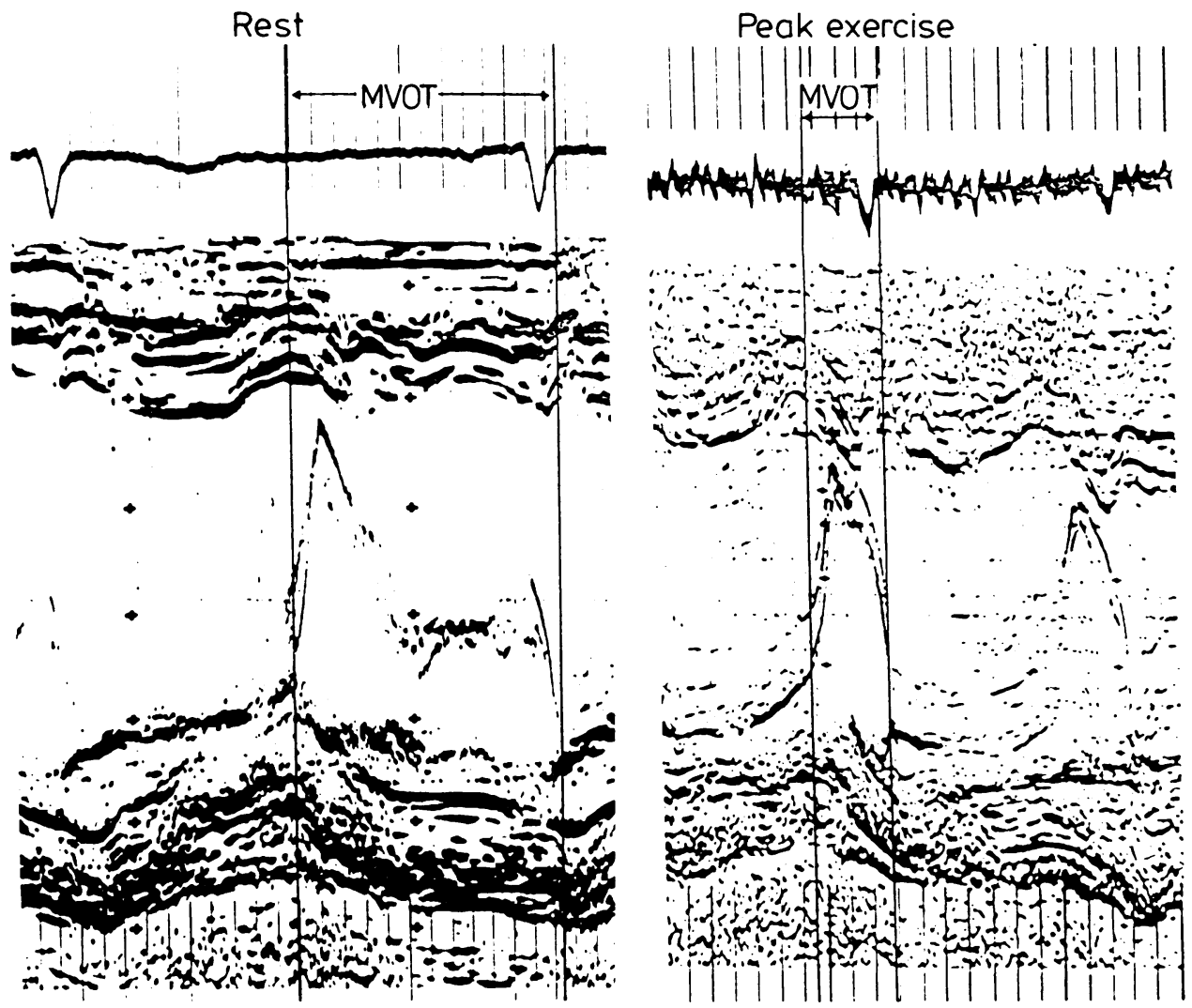

Fig. 1 Typical record showing mitral valve motion at peak exercise in a normal subject. 
(4) The interval A2 to mitral valve opening (isovolumic relaxation time), the latter being taken as the time of initial separation of the leaflets at the onset of ventricular filling. ${ }^{4}$

(5) Total left ventricular filling time, measured as the interval from mitral valve opening to closure at the start of the succeeding ventricular systole, expressed in $\mathrm{ms} / \mathrm{beat}$. The relative time available for left ventricular filling was also calculated as the product of the duration of mitral valve opening and heart rate, representing the total time that the mitral valve was open during each minute, with the units in $\mathrm{s} / \mathrm{min}$.

(6) Resting records were digitised, ${ }^{5}$ and peak systolic and diastolic rates of dimension change were computed.

All measurements were derived as the mean value from three beats. Mean values of different groups were compared using Student's t test. Linear regression was performed by the method of least squares.

\section{Results}

\section{RESTING VALUES}

All patients studied after aortic valve replacement had evidence of left ventricular hypertrophy, with mean values of posterior wall and septal thickness, measured at end-diastole, being $1.4 \pm 0.3$, and $1.6 \pm 0.4 \mathrm{~cm}$, respectively. Left ventricular cavity size was within normal limits at end-systole $(3.6 \pm 0.7 \mathrm{~cm})$ and enddiastole $(4.6 \pm 0.9 \mathrm{~cm})$. Systolic function, expressed as peak VCF, was normal in all but two patients, with a mean value of $1.8 \pm 0.7 / \mathrm{s}$. Diastolic function, however, was abnormal with isovolumic relaxation time prolonged to $85 \pm 8 \mathrm{~ms}$ (normal $69 \pm 2 \mathrm{~ms}, \mathrm{p}<0.01$ ), and peak rate of dimension increase during filling reduced to $11.6 \pm 4 \mathrm{~cm} / \mathrm{s}$ (normal $16 \pm 3 \mathrm{~cm} / \mathrm{s}, \mathrm{p}<0.01)$. These findings are characteristic of secondary left ventricular hypertrophy. ${ }^{5}$ In the normal subjects at rest, the mitral valve was open during diastole for a mean period of $380 \pm 66 \mathrm{~ms} /$ beat, or $27 \pm 2 \mathrm{~s} / \mathrm{min}$. Corresponding values in the patients were not significantly different, being $367 \pm 66 \mathrm{~ms} /$ beat or $27 \pm 3 \mathrm{~s} / \mathrm{min}$.

\section{SYSTOLIC FUNCTION DURING EXERCISE}

In normal subjects, end-diastolic dimension increased by $2 \mathrm{~mm}$ ( $p<0.01$ with respect to resting values) and end-systolic dimension fell by $1 \mathrm{~mm}(p<0.05)$. Mean shortening fraction increased by $6 \%$, and peak VCF from 2.6 to $3.6 / \mathrm{s}$ (both $\mathrm{p}<0.01$ ). Changes in the patients were very similar: end-diastolic dimension increased by a mean of $1.5 \mathrm{~mm}(\mathrm{p}<0.01)$, and endsystolic dimension fell by $0.5 \mathrm{~mm}$ (not significant). Shortening fraction increased from 24 to $27 \%$ $(\mathbf{p}<0.01)$, and peak VCF from 1.8 to $2.5 / \mathrm{s}(\mathbf{p}<0.01)$.
The effect of increasing heart rate on QA2 interval $c$. was given by the regression equation:

$$
\mathrm{QA2}=-215+2 \cdot 53 \mathrm{RR} \text {, }
$$

where $R R$ represents $R R$ interval. The correlation coefficient was 0.86 and the standard error of the $\frac{\bar{m}}{\frac{9}{2}}$ estimate $82 \mathrm{~ms}$. The corresponding relation for the $\stackrel{\mathbb{Q}}{\Omega}$ normal subjects was:

$$
\mathrm{QA2}=-202+2.67 \mathrm{RR} \text {, }
$$

with a correlation coefficient of 0.97 and a standard error of the estimate of $45 \mathrm{~ms}$. There was no significant difference between either the slopes or the intercepts of these two relations. Values for the rela-? tion between RR and QA2 intervals for the five $\overrightarrow{0}$ patients during the atrial pacing were not significantly ${ }^{\circ}$ different from the above.

\section{DIASTOLIC FUNCTION DURING EXERCISE}

Isovolumic relaxation time
In normal subjects, the duration of isovolumic relaxation time remained virtually constant with exercise for rates up to 140 to $150 \mathrm{bpm}$, the mean value at peak $\vec{\infty}_{\infty}$ recorded levels being $70 \pm 9 \mathrm{~ms}$, which is not significantly different from the resting value. Though isovolumic relaxation time was longer than the normals at rest in the patients with an aortic valve replace- $\frac{\partial}{0}$ ment, it fell consistently with exercise, so that at the highest levels studied it had fallen to $41 \pm 15 \mathrm{~ms} \underset{\complement}{\mathbb{\Phi}}$ significantly less than normal $(p<0.01)$. This relation is shown in Fig. 2. The main reduction in isovolumiç relaxation time occurred as heart rate increased from 70 to 100 beats per minute; it then remained virtually constant at higher exercise levels in all but one patient in whom it dropped to the very low value of $10 \mathrm{~ms}$.

\section{Duration of left ventricular filling period}

In normal subjects, the duration of the left ventriculat filling period progressively dropped, so that at max $\frac{0}{3}$ imum levels at which recordings could be made, the mitral valve was open for $115 \pm 10 \mathrm{~ms} /$ beat, or $16 \pm 2 \mathrm{~s} / \mathrm{min}$. Its behaviour in the patients was very different from normal. At maximum levels recorded the duration of mitral valve opening had dropped too $240 \pm 44 \mathrm{~ms} /$ beat, significantly greater than the cor:responding value in the normal group $(\mathbf{p}<0.01)$ (Fig $3)$. When expressed as seconds per minute, left ven $\omega$ tricular filling period remained unchanged during exercise at $28 \pm 4$ in the patients, the response agaim being significantly different from normal $(p<0.001$ 跑 (Fig. 4).

\section{Atrial pacing}

The patients were studied at rest, and at a rate of 150 beats/min. At rest, isovolumic relaxation time was normal $(66 \pm 7 \mathrm{~ms})$, and was effectively unaltere $\$$ by pacing $(62 \pm 10 \mathrm{~ms})$. Left ventricular filling time 


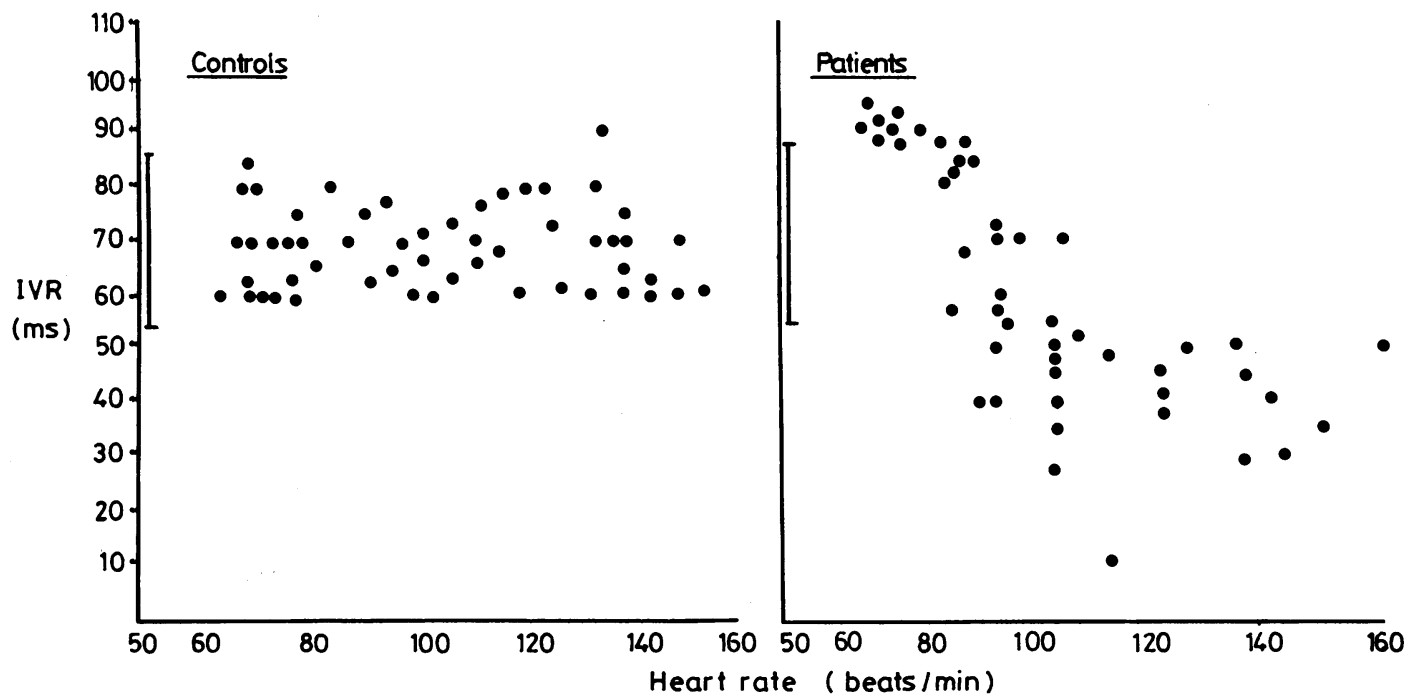

Fig. 2 Relation between heart rate and the duration of isovolumic relaxation time in controls and patients after aortic valve replacement.

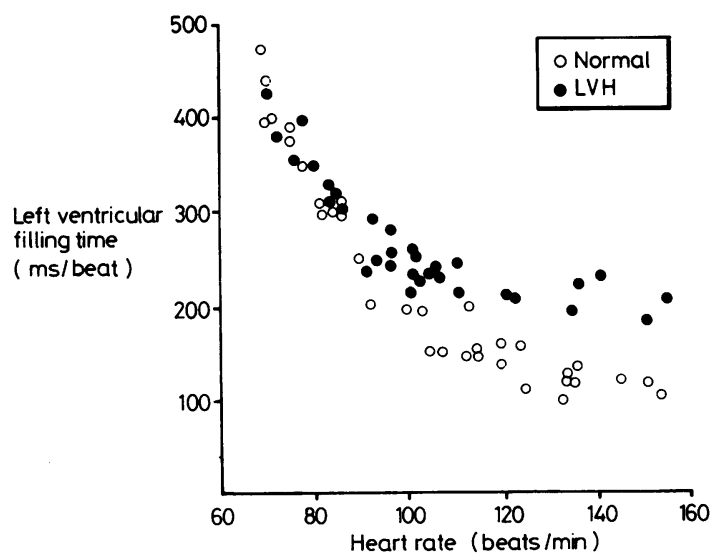

Fig. 3 Relation between heart rate and left ventricular filling time, expressed in ms/beat. Note the separation between patients and normal subjects.

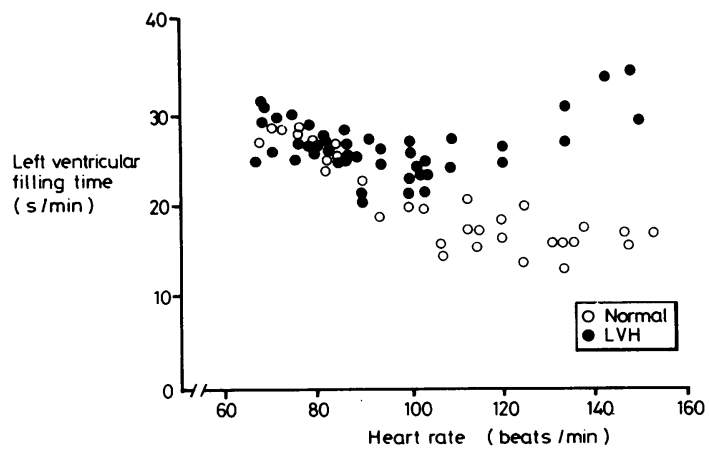

Fig. 4 Relation between heart rate and left ventricular filling time, expressed in s/min. Note that there is no significant fall in this measurement in the patients, a response that is significantly different from normal. was normal at rest, being $344 \pm 36 \mathrm{~ms} /$ beat dropping to $108 \pm 13 \mathrm{~ms}$ during pacing. This latter value was not significantly different from that recorded during exercise in the normal subjects at the same heart rate. At rest, the mitral valve was open $26 \pm 1 \mathrm{~s} / \mathrm{min}$, and during artial pacing this dropped to $16 \pm 2 \mathrm{~s} / \mathrm{min}$, again not significantly different from normal subjects at peak recorded exercise level.

\section{Discussion}

In the present investigation, two phases of diastole were defined during exercise; isovolumic relaxation and left ventricular filling period as the time the mitral valve was open. These could be measured by a simple combination of phono- and echocardiography, which proved to be technically feasible in the majority of patients. The method differs from the diastolic interval previously described by Weisdorf and Spodick, ${ }^{8}$ in that left ventricular filling period was measured directly, so that abnormal prolongation and possible variation in isovolumic relaxation time with heart rate could be allowed for.

Adaptation to exercise in normal subjects has been extensively studied. ${ }^{910}$ Except at low exercise levels, the increase in cardiac output in untrained individuals is brought about largely by a change in heart rate, with only a minor increase in stroke volume. Though ejection time shortens, the positive inotropic effect of increased sympathetic activity and the reduction in peripheral resistance allow ejection rate to rise and stroke volume to be maintained. Changes in ventricular relaxation and filling on exercise, however, have attracted little interest. In normal subjects, a clear pattern was seen. There was no change in isovolumic 
relaxation time up to a heart rate of $150 / \mathrm{min}$. By contrast, left ventricular filling time became progressively shorter, consistently falling to just above $100 \mathrm{~ms} /$ beat at a rate of $150 / \mathrm{min}$. Since stroke volume was likely to have remained virtually constant or even to have increased slightly at this level of exercise, the results suggest a progressive rise in mean ventricular filling rate, compatible with observations using nuclear angiography. ${ }^{3}$ This shortening of the filling period was not related to any change in left ventricular inotropic state, since a virtually identical relation to heart rate was seen during atrial pacing. We conclude therefore that in the normal subject, left ventricular filling time is a simple function of heart rate, while isovolumic relaxation time is virtually independent of it.

The possible value of studying events during early relaxation using the present method was shown in patients with left ventricular hypertrophy. In all but two of these patients, systolic function at rest was normal, whether judged in terms of changes in cavity size, shortening fraction, peak VCF, or QA2 interval. In patients with secondary left ventricular hypertrophy, isovolumic relaxation time was considerably prolonged at rest. ${ }^{4}$ Indeed, values in excess of $150 \mathrm{~ms}$ may be found in such patients, considerably longer than the entire filling period in normal subjects at relatively low exercise levels. It might be anticipated, therefore, that diastolic events would have to change considerably during exercise in patients with left ventricular hypertrophy if ventricular filling were to be achieved at all. The present results shed light on this question. During exercise in patients with left ventricular hypertrophy, there was a striking reduction in isovolumic relaxation time, so that, from having been significantly longer than normal at rest, it became significantly shorter than normal on exercise. This fall in isovolumic relaxation time occurred at relatively low exercise levels, and thereafter stabilised in the majority of patients even when there was a further increase in heart rate. The duration of mitral valve opening also fell with exercise, but not to the low levels seen in normal subjects, so that there was no fall in the total time per minute that the mitral valve was open. The difference between normal and abnormal was considerable, being a factor of approximately two in the present study, implying that the maximum velocity of ventricular filling must have been very significantly reduced on exercise in patients with left ventricular hypertrophy.

This ability to achieve mean filling rates considerably in excess of those during ejection is presumably required to maintain stroke volume during exercise, and presupposes complex underlying mechanisms. These are likely to include ventricular filling as pressure is falling, ${ }^{6}$ and the presence of early diastolic restoring forces generated during the previous sys- $c$ tole. ${ }^{11}$ Passive stress-strain relations are probably not significant ${ }^{12}$ at fast heart rates, and neither is the ino $\stackrel{-5}{\rightarrow}$ tropic state of the left ventricle involved. Another? potential mechanism for maintaining diastolic filling, that of prolonged filling period by abbreviating? isovolumic relaxation time, was clearly excluded in normal subjects.

By contrast, in patients with left ventricular hyper- $\vec{\theta}$ trophy, isovolumic relaxation time progressivelyshortened with exercise. This occurred at lower heart $\vec{\omega}$ rates, when the left ventricular filling time was stillo within the normal range. The underlying mechanism? for this shortening is uncertain, but one possibility iste an increase in left atrial and left ventricular end-os diastolic pressures. ${ }^{13}$ Again, it is unlikely to have beenor simply a manifestation of increased sympathetic activ- $-\infty$ ity, which also presumably occurred in normal sub-? jects during exercise, without a change in isovolumic relaxation time. The complete separation of the patients from the normal subjects, and the relative ${ }^{D}$ independence of rate at values greater than $120 / \mathrm{min}, \overrightarrow{0}$ both seem to suggest that functional loss in these ven-w tricles was uniform and complete, allowing the notable contribution of the underlying mechanisms to normal filling to be assessed. In spite of these striking diastolic abnormalities, systolic function was pre-served during exercise as it was previously noted too have been at rest. Since many of these patients have severely impaired exercise tolerance in spite of norma $\overline{\mathrm{Q}}$ cavity size and peak VCF, ${ }^{14}$ a limiting factor may well be abnormal diastolic function and, in particular, fail ure to achieve rapid ventricular filling rates when:heart rate is high.

Our results indicate that fundamental information about left ventricular diastolic function can beo obtained during exercise from simple echocardiog raphic measurements, allowing the requisite informa- tion to be collected at relatively high exercise levels. In particular, it seems to be easier to record mitraf valve motion during exercise than to obtain satisfac- $\rightarrow$ tory measurements of left ventricular cavity size. Since the difference between normal and abnormal is so large, these methods may prove sensitive in detect- ing less obvious disturbances of diastolic function $\tilde{O}$ whose potential clinical significance is, as yet, unre $-\widetilde{\omega}$ cognised, and even be used as the basis of a simple stress test with wide application in clinical practice.

\section{References}

1 Mirsky I. Assessment of passive elastic stiffness of car $\frac{\rho}{\mathbb{D}}$ diac muscle: mathematical concepts, physiologic andळ clinical considerations, directions of future research. Prog Cardiovasc Dis 1976; 18: 277-308. 
2 Gibson DG, Traill TA, Hall RJC, Brown DJ. Echocardiographic features of secondary left ventricular hypertrophy. Br Heart F 1979; 41: 54-9.

3 Bonow RO, Bacharach SL, Green MV, et al. Impaired left ventricular diastolic filling in patients with coronary artery disease: assessment with radionuclide angiography. Circulation 1981; 64: 315-23.

4 Chen W, Gibson D. Relation of isovolumic relaxation to left ventricular wall movement in man. Br Heart $\mathcal{F}$ 1979; 42: $51-6$.

5 Upton MT, Gibson DG. The study of left ventricular function from digitized echocardiograms. Prog Cardiovasc Dis 1978; 20: 359-84.

$6 \mathrm{Katz} \mathrm{LN}$. The role played by the relaxation process in filling of the ventricle. Am F Physiol 1930; 95: 542-53.

7 Corallo S, Broso GP, Seaga R, et al. Value and limitations of exercise echocardiography in normal and pathological conditions. In: Kurjak A, Kratochwill A, eds. Recent advances in ultrasound diagnosis: no. 3. Amsterdam: Excerpta Medica, 1981: 428-32.

8 Weisdorf D, Spodick DH. Duration of diastole versus cycle length as correlates of left ventricular ejection time. Br Heart F 1976; 38: 282-4.

9 Astrand PO, Cuddy TE, Saltin B, Stenberg J. Cardiac output during submaximal and maximal work. $\mathcal{F}$ Appl Physiol 1964; 19: 268-74.

10 Blomquist CG. Exercise physiology; clinical aspects. In: Wenger NK, ed. Exercise and the heart. Philadelphia: FA Davis, 1978: 1-12.

11 Sonnenblick EH. The structural basis and importance of restoring forces and elastic recoil for the filling of the heart. Eur Heart f 1980; 1: A-107-10.

12 Traill TA, Gibson DG. Left ventricular relaxation and filling: study by echocardiography. In: Yu PN, Goodwin JF, eds. Progress in cardiology, 8. Philadelphia: Lea and Febiger, 1979: 39-72.

13 Mattheos M, Shapiro E, Oldershaw PJ, Sacchetti R, Gibson DG. Non-invasive assessment of changes in left ventricular relaxation by combined phono-, echo-, and mechanocardiography. Br Heart $\mathcal{f}$ 1982; 47: 253-60.

14 Dawkins K, Cotter L, Gibson D. Limitations of the New York Heart Association criteria in the assessment of exercise tolerance after aortic valve replacement. Circulation 1981; 64 (suppl IV): 259.

Requests for reprints to Dr P J Oldershaw, Brompton Hospital, Fulham Road, London SW3 6HP. 